\title{
Role of Hole Localization in the Magneto-Luminescence of a Two-Dimensional Electron Gas
}

\author{
J.M. Calleja, D. Sarkar, H.P. van der Meulen
}

Dept. Física de Materiales, Universidad Autónoma de Madrid

Cantoblanco, 28049 Madrid, Spain

\author{
R. Hey, K.J. Friedland and K. Ploog
}

Paul-Drude Institut für Festkörperelektronik

Hausvogteiplatz 5-7, 10117 Berlin, Germany

We investigate the effect of hole localization on the magneto-luminescence emission of a two-dimensional electron system formed in a modulation-doped semiconductor heterojunction. The emission of heterojunctions containing a dilute delta-layer of Be acceptors at a large distance from the interface is compared with that of Be-free samples. A narrow Fermi-edge singularity emission line is observed at low temperature in Be-doped samples, involving electrons in the second confined state. The evolution of this line in a perpendicular magnetic field shows common characteristics with previously reported results in high mobility samples not containing Be. A new emission line appears abruptly at filling factor 2 originating by the recombination of electrons in the lowest Landau level with free valence holes, independent of the presence of Be. The abruptness of this transition, which is also observed in some samples at filling factor 1, reveals a simultaneous change in the electron system over a macroscopic sample area. The new optical emission shows marked deviations with respect to the single-particle behavior, which are tentatively interpreted as the formation of a complex state involving a free photocreated hole and the electron system. This complex unbinds when the Fermi level crosses the mobility edge.

PACS numbers: 73.43.Lp, 78.55.Cr, 73.21.-b

\section{Introduction}

Optical measurements on the high mobility two-dimensional electron system (2DES) have been performed over a long period [1-13] to study electron interactions through their effect on the optical emission properties. Many-body effects 
have an influence on the optical spectra, as revealed by the appearance of charged excitons at low electron densities [11], shake-up processes [12], and optical singularities at the Fermi level (FES) [13]. Most of the experimental work on optical emission by a 2DES has been done in the GaAs-AlGaAs system, due to the high electron mobility obtained at its interface. The interaction effects are strongly dependent on carrier localization and on the screening properties of the electron gas. In a perpendicular magnetic field, the screening properties change as the 2DES passes through different filling factors when the field is varied, giving alternating states where the Landau levels are completely filled (incompressible) or partially filled (compressible). Thus, the magneto-emission is sensitive to the compressibility of the electron system. The presence of the photocreated holes in photoluminescence (PL) experiments can perturb the 2DES properties. This is often the main drawback of optical methods compared to transport measurements. One way to minimize this perturbation is to use a modulation-doped semiconductor heterojunction (HJ) [1-8], instead of a quantum well (QW) $[9,10]$ where the electrons and holes are close to each other. In HJs the photocreated holes tend to escape away from the interface due to the built-in electric field. The PL emission from the 2DES then vanishes unless the hole is kept near the interface. This can be achieved either by trapping at uncontrolled interface defects, by screening of the electric field (band flattening) due to residual doping [1] or by trapping of the photocreated hole $(\mathrm{PCH})$ at a $\delta$-layer of acceptors (normally Be) located at a certain distance from the HJ $[2-5,8]$. This last method has been widely used to investigate the 2DES in the integer [3, 8] and fractional $[2,4]$ quantum Hall regimes. A large variety of magneto-photoluminescence (MPL) characteristics of the 2DES have been reported in the past, which depend strongly on the sample structure and details. Simultaneous measurement of MPL and Hall resistivity in GaAs QWs [3, 9] and HJs [1, 7, 8] have revealed deviations of the dependence of the emission energy on magnetic field with respect to the normal Landau-level behavior near $\nu=1$ and 2, as well as changes in the emission intensity. The reported energy deviations occur at higher or lower energies depending on the well width and have been interpreted as the result of a magnetic-field induced change in the screening of the PCH by the electron gas [14]. Also, a splitting of the PL emission of a 2DES around odd filling factors has been reported in GaAs quantum wells. It has been attributed to the interference of the photocreated hole with the continuum of spin-wave excitations of the Fermi sea [10]. MPL emission in HJs has been observed mainly for localized holes, but in some cases emission involving free holes has also been reported $[1,5-7]$. The PL emission in this case shows marked deviations from the single-particle trend at integer filling factors. For recombination with free holes one expects conservation of the Landau level (LL) index. In this case and assuming low power excitation, the few photocreated holes relax quickly to their ground state, and hence only recombination with electrons in the lowest LL is possible. 
In this paper we investigate the role of hole localization in the MPL response of a 2DES in a single semiconductor HJ. For this purpose we compare the emission spectra of samples having a dilute delta-doping layer of Be acceptors at a large distance from the HJ interface with the emission of Be-free samples. The two types of samples have similar electron densities and mobilities, with two electron states confined by the HJ potential. The Fermi level is slightly above the second confined state, so that its electron density is very low. Compared to previous work [2-4], the distance of the Be layer from the HJ in the Be-doped samples is large enough to prevent overlap between the electrons in the lowest confined state and the photocreated hole wave functions. In Be-doped samples, the simultaneous measurement of MPL and longitudinal and transverse resistivity reveals sharp peaks in the MPL spectrum at integer filling factors, which are due to Fermi-edge singularities associated with electrons in the weakly populated second confined sub-band recombining with localized holes [8]. The temperature dependence of these sharp peaks indicates differences in the suppression of screening at even and odd filling factors. The dependence of the intensity of this emission on magnetic field coincides strikingly with data reported for a high-mobility, low-density 2DES [1], thus indicating that hole localization by residual disorder can play a key role even in very high mobility samples.

For both kinds of samples the abrupt onset of a new MPL emission occurs below filling factor $\nu=2$, involving free valence band holes. In Be-doped samples, it coincides with the equally abrupt disappearance of the Be-bound-hole luminescence. At low temperatures, the dependence of the energy and intensity of the new emission on the magnetic field deviates significantly from the standard Landau-level behavior, as long as the electron system is in an incompressible state. A similar behavior is observed in some samples at $\nu=1$. This behavior is tentatively attributed to the formation of a complex state involving a small number of LL-holes (electrons missing in one of the spin branches of the lowest LL) and the valence band hole.

\section{Experiment}

The $\mathrm{Al}_{x} \mathrm{Ga}_{1-x} \mathrm{As} / \mathrm{GaAs}$ modulation doped single HJs are grown for $x=0.33$ by molecular beam epitaxy on GaAs (001) substrates. The samples consist of a GaAs/AlGaAs short-period superlattice buffer layer, a $1000 \mathrm{~nm}$ thick GaAs layer, an undoped AlGaAs spacer layer including a Si doped layer, and a thin undoped GaAs cap layer. In one of the samples (sample $A$ ) a dilute Be $\delta$-doping layer $\left(1 \times 10^{9} \mathrm{~cm}^{-2}\right)$ is located in the GaAs side at a distance $d=60 \mathrm{~nm}$ from the HJ interface. The electron density of sample $A$ is $n_{A}=3.16 \times 10^{11} \mathrm{~cm}^{-2}$, corresponding to a Fermi energy $E_{\mathrm{F}}=11.3 \mathrm{meV}$. The other samples (samples $B$ and $C$ ) have no Be layer. Their electron densities are $n_{B}=3.24 \times 10^{11} \mathrm{~cm}^{-2}$ and $n_{C}=1.96 \times 10^{11} \mathrm{~cm}^{-2}$ respectively. The electron mobilities lie between 


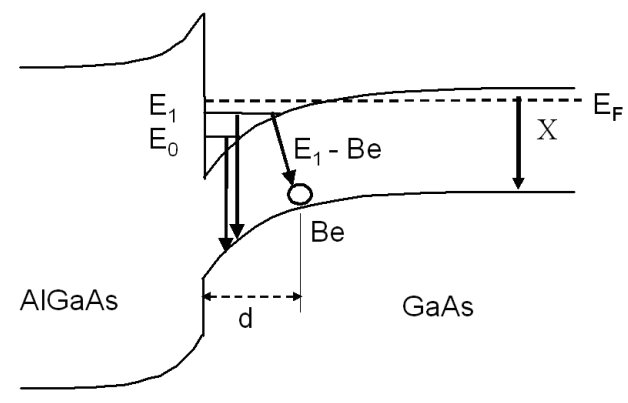

Fig. 1. Energy band diagram for the samples showing the confined electron states and the optical transitions.

1 and $2 \times 10^{6} \mathrm{~cm}^{2} /(\mathrm{V} \mathrm{s})$. The band structure diagram is presented in Fig. 1, showing two confined electron states $\left(E_{0}, E_{1}\right)$ with the Fermi level $E_{\mathrm{F}}$ slightly above $E_{1}$. The arrows indicate the PL transitions discussed in the next section. Patterning of either a Hall bar or a rectangular area with lateral contacts on some of the samples allowed simultaneous measurement of PL and transport properties. PL measurements were performed at different temperatures down to $280 \mathrm{mK}$ in a ${ }^{3} \mathrm{He}$-immersion insert in a superconducting magnet providing magnetic fields up to $12 \mathrm{~T}$. The samples were accessed optically through windows located at the bottom of the magnet cryostat. A Ti-sapphire laser was used for PL excitation below the band gap of the AlGaAs barrier. Great care was taken to screen out higher energy light for simultaneous measurements of PL and transport. The diameter of the laser spot on the sample was around 100 micrometers. The excitation power was kept below $30 \mathrm{~mW} / \mathrm{cm}^{2}$, and the emitted light was detected with a double spectrometer and a charge-coupled detector. All the measurements were performed at $300 \mathrm{mK}$, unless otherwise specified.

\section{Results and discussion}

The emission spectrum of sample $A$ is shown in Fig. 2 for zero magnetic field on a logarithmic ordinate scale. The main peak at $1.514 \mathrm{eV}$ is mainly due to exciton recombination in the thick GaAs layer. A weak step at $1.505 \mathrm{eV}$ corresponds to the recombination of $E_{0}$ electrons with photocreated holes, probably localized at uncontrolled shallow interface defects. A similar transition occurs involving $E_{1}$ electrons, whose energy accidentally coincides with the excitonic emission. This is proved by the existence of a clear shake-up emission in the MPL spectra, whose energy extrapolates to $1.514 \mathrm{eV}$ at zero magnetic field. At low energies, the sharp peak at $1.491 \mathrm{eV}$ comes from $E_{1}$ electrons recombining with photocreated holes trapped at the Be layer. The broad band below it is due to donor-acceptor transitions related to carbon impurities. The energy difference between the two $E_{1}$ emissions is $23 \mathrm{meV}$, which is slightly below the Be binding energy in GaAs: 


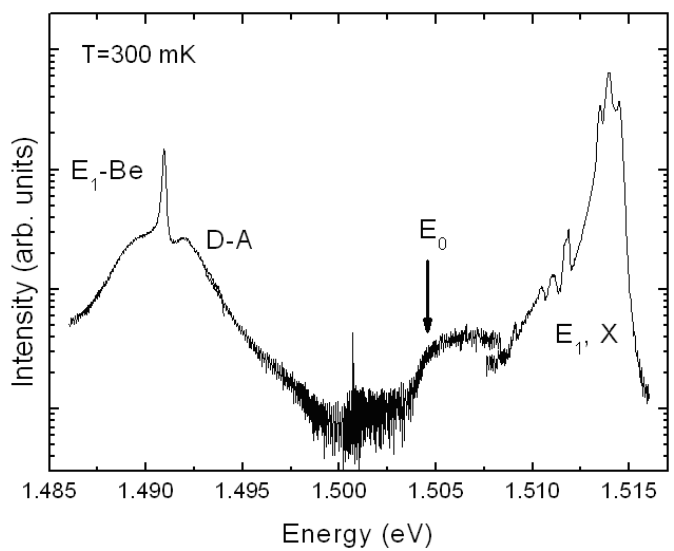

Fig. 2. Photoluminescence spectrum of sample $A$ at zero magnetic field and $300 \mathrm{mK}$. The labels of the different peaks correspond to the transitions indicated in Fig. 1 and are explained in the text. The broad emission D-A is due to donor-acceptor transitions corresponding to carbon impurities.

$28 \mathrm{meV}$ [15]. The difference is due to the band bending near the HJ. No traces of $E_{0}$ electron recombination with Be-trapped holes have been observed. This indicates that the wave function extension of $E_{0}$ electrons away from the interface is less than $60 \mathrm{~nm}$. For $E_{1}$ electrons this extension can reach $100 \mathrm{~nm}$ [8]. The intensity of the sharp $E_{1}$-Be emission decreases very rapidly with increasing temperature (it reduces to one half from $300 \mathrm{mK}$ to $3 \mathrm{~K}$ ). It is interpreted as an optical FES involving $E_{1}$ electrons and the localized PCH. A similar FES has been reported in Ref. [3], where both $E_{0}$ and $E_{1}$ electrons recombine with photocreated holes localized at Be acceptors.

The dependence of the energy (ordinate scale) and intensity (gray scale) of the $E_{1}$-Be emission on the magnetic field is shown in Fig. 3. Between zero and $0.2 \mathrm{~T}$, the energy remains constant. At higher fields it follows a linear trend indicating LL behavior. The magnetic length corresponding to $0.2 \mathrm{~T}$ is $57 \mathrm{~nm}$, so that the constant PL energy at low field can be interpreted as due to electron scattering by residual disorder with a length scale of this magnitude. The value of $0.2 \mathrm{~T}$ is also found in a sample where the Be is $100 \mathrm{~nm}$ from the HJ. Consequently this scattering length is not related to the Be-HJ separation. Also, the average inter-Be separation is of the order of $300 \mathrm{~nm}$, so that Be acceptors are not responsible for this disorder. The slope of the linear evolution at higher fields is $0.89 \mathrm{meV} / \mathrm{T}$, corresponding to the conduction electron effective mass*. One observes intensity oscillations with pronounced maxima at integer values of the filling factor $(\nu)$. In the regions between maxima at high magnetic field, the PL intensity is rather field-independent. The field-dependent population of the $E_{1}$

${ }^{*}$ The Zeeman splitting of the Be levels is twenty times smaller, as shown in [16]. 


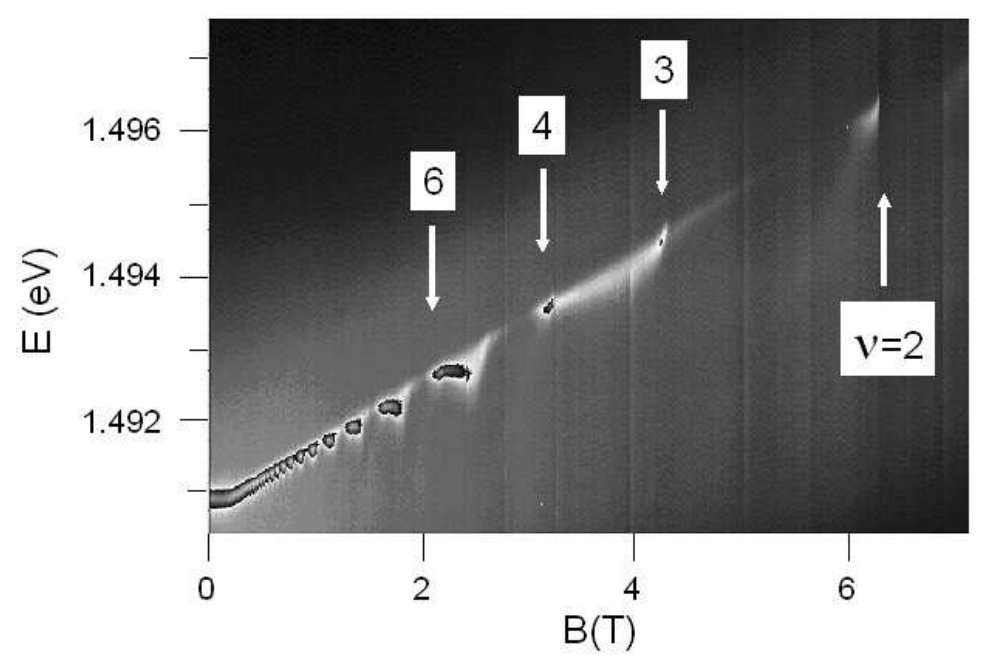

Fig. 3. Energy (ordinate scale) and intensity (gray scale) of the $E_{1}$-Be emission of sample $A$ versus applied magnetic field. Relevant filling factors are indicated. The slope of the linear energy change corresponds to free electrons and localized holes.

confined state is not sufficient to explain the MPL behavior observed. In a simple minded single-particle picture this population increases progressively with the field from every crossing of an $E_{0}$ LL with the lowest $E_{1}$-LL until the next even filling factor is reached. There, as shown in Fig. 4, the Fermi energy (more properly: the chemical potential) drops again to the next lower $E_{0}$-LL. This picture is not essentially modified in a self-consistent calculation including field-induced changes in the HJ potential [3]. The expected MPL intensity dependence should show periodic abrupt changes at even filling factors and continuous changes in between, as reported in Ref. [3]. Instead, the sharp peaks reported here appear to be due

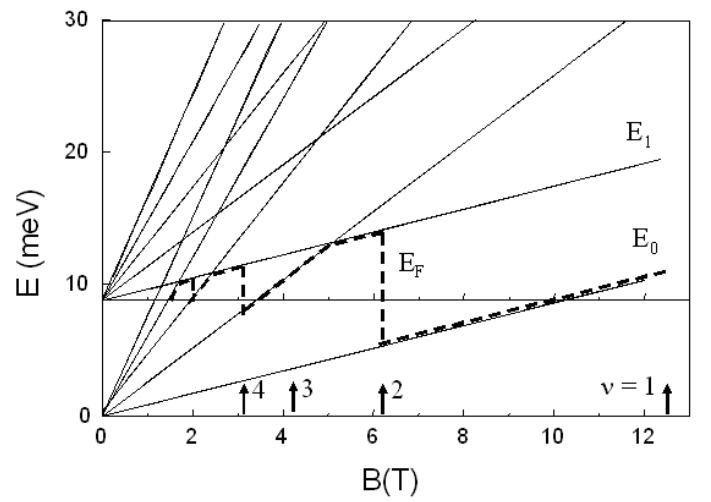

Fig. 4. Fan diagram for sample $A$ showing the LL of the $E_{0}$ and $E_{1}$ states and the alternation of the Fermi energy between them as the magnetic field changes. 
to the combined effects of $E_{1}$ population and the FES enhancement, as observed at zero field. Sharp peaks occur also at odd filling factors, as seen at $\nu=3$ in Fig. 3, where the $E_{1}$ population is not expected to change significantly. The origin of the sharp peaks in the MPL intensity seems to be linked to suppression of the screening by the $2 \mathrm{DES}$. Although $E_{0}$ electrons cannot recombine with Be-trapped holes because of lack of wave function overlap, they can screen the Be potential when they are mobile. Thus, when the system is incompressible the Be potential cannot be screened by $E_{0}$ electrons and the $E_{1}$-Be FES appears. These MPL results are surprisingly similar to those reported in Ref. [1] for a high mobility $\left(9 \times 10^{6} \mathrm{~cm}^{2} /(\mathrm{V} \mathrm{s})\right) 2 \mathrm{DES}$ in a Be-free HJ. One has to conclude that $E_{1} \mathrm{MPL}$ emission in Ref. [1] is due to hole localization at residual disorder, in spite of the high mobility of the sample.

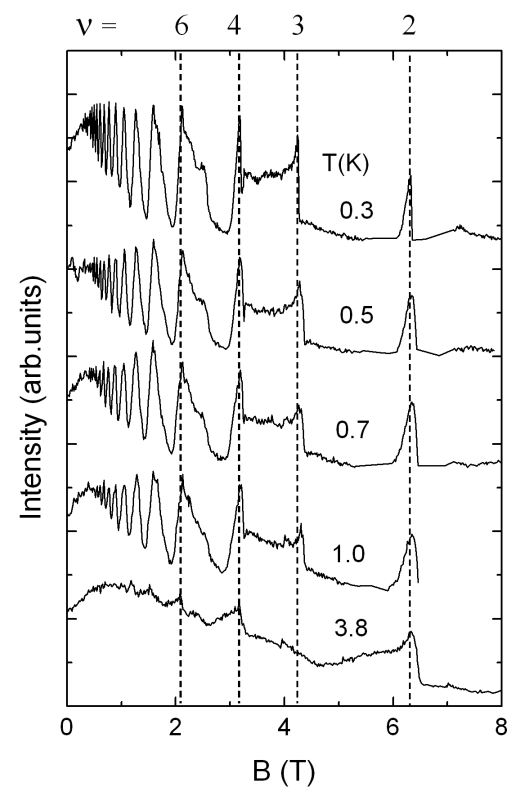

Fig. 5. Temperature and magnetic field dependence of the $E_{1}$-Be emission of sample $A$.

The intensity of the $E_{1}$-Be MPL and its temperature dependence are shown in Fig. 5. We observe rapid intensity decrease in the peaks at even $\nu$ as the temperature increases. The decrease is similar to the one observed at zero field. Consequently, it reflects the typical FES behavior rather than the thermally enhanced screening of the Be potential. The sharpness of the peaks indicates that the transition across integer filling occurs simultaneously over the whole sample area illuminated by the excitation beam. The peak at $\nu=3$ decreases faster than those at even filling factors when the temperature increases. A possible explanation of this difference is the small Zeeman splitting at $4.2 \mathrm{~T}(0.1 \mathrm{meV})$, which determines 
the jump in the chemical potential at $\nu=3$, compared with the inter-LL jump at even filling factors 2 and 4 , given by the cyclotron energy (see Fig. 4).

At $\nu=2$ there is an abrupt transfer of the $E_{1}$-Be intensity to a new emission line at $1.496 \mathrm{eV}$, similar to that observed in Be-free samples [7]. In Fig. 6 the waterfall plots of the MPL spectra of samples $A$ and $B$ are shown. The magnetic field scale for sample $B$ is slightly different from that for sample $A$ (shown in the figure) to make the filling factors coincide in the two samples. For sample $A$ two different spectral regions display the $E_{1}$-Be emission (left) and the $E_{0}$ one (right). For both samples the abrupt onset of the luminescence from the lowest $E_{0}$-LL at $\nu=2$ is observed ( $\nu=2$ is marked by a white dashed line). A similar onset,
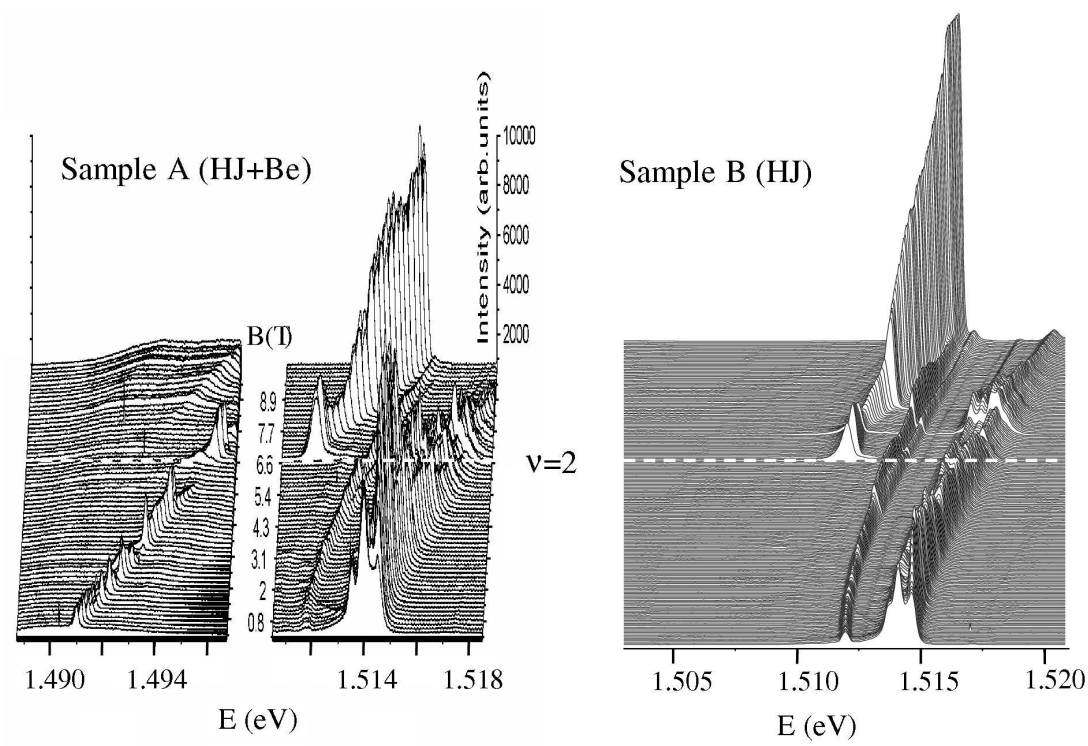

Fig. 6. Waterfall plots of samples $A$ (left) and $B$ (right) showing the evolution of the spectra and the abrupt onset of the $E_{0}$ emission at $\nu=2$. The magnetic field scale of sample $B$ has been slightly changed according to the difference of the electron densities, in order to display a common filling factor scale.

although not abrupt, has been reported in Ref. [5]. The linear field dependence of this emission extrapolates to the $E_{0}$ shoulder in Fig. 2 with a slope indicating a reduced mass of 0.055 . This corresponds to both free electrons $\left(m_{\mathrm{e}}^{*}=0.067\right)$ and free valence-band holes $\left(m_{\mathrm{h}}^{*}=0.34\right)^{\dagger}$. A similar result is observed in Be-free samples, where the lowest $E_{0}$-LL emission is weakly observed also between filling factors 3 and 4 [7]. The strong $E_{0}$ emission below $\nu=2$ requires a "binding" of the valence-band hole to the $2 \mathrm{DES}$, which is strong enough to suppress trapping at $\mathrm{Be}$ acceptors in sample $A$. The binding cannot be due to defects or interface roughness

${ }^{\dagger} m_{\mathrm{h}}^{*}=0.34$ is the reported value for the triangular confining potential of a HJ [17]. 
as in the case of the $E_{0}$ emission at zero field, as the hole is extended. Rather it must come from the formation of a many-body complex involving the 2DES and the valence hole. Below $\nu=2$ the $E_{0}$ energy deviates from the linear LL trend in both samples until around $\nu=1.7$. At higher fields, the standard behavior is recovered. The details of the "anomalous" behavior are sample-dependent, but the main characteristics (red shift with respect to the LL line and kink with minimum intensity around $\nu=1.7$ ) are reproducible. The simultaneous measurement of the $E_{0}$ emission and the longitudinal and transverse resistance of the samples indicates clearly that the $E_{0}$ emission onset corresponds to $\nu=2$, and the kink coincides with the crossing of the chemical potential with the mobility edge of the 2DES. This is shown in Fig. 7 for sample $C$. The upper panel of Fig. 7 shows

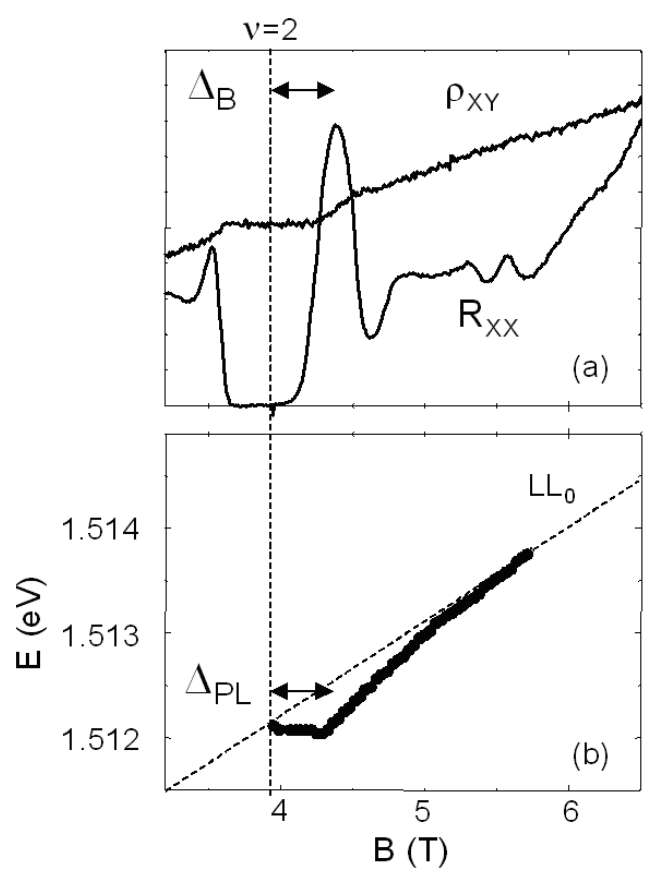

Fig. 7. Detail of the simultaneous transport (a) and optical (b) measurements around $\nu=2$ in sample $C$. The longitudinal resistance plateau $\Delta_{B}$ and the optical "anomaly" $\Delta_{\mathrm{PL}}$ are indicated. The oblique dashed line represents the "standard" LL trend.

the $R_{x x}$ and $\rho_{x y}$ traces near $\nu=2$, while the lower one displays the $E_{0}$ MPL energy versus magnetic field. The oblique dashed line represents the linear LL trend. $\Delta_{\mathrm{PL}}$ indicates the field range of the optical "anomaly" and $\Delta_{B}$ marks the mobility edge. The temperature dependences of $\Delta_{\mathrm{PL}}$ and $\Delta_{B}$ shown in Fig. 8 are indistinguishable from each other. This proves the direct link between the optical anomalies reported and the compressibility of the 2DES. 


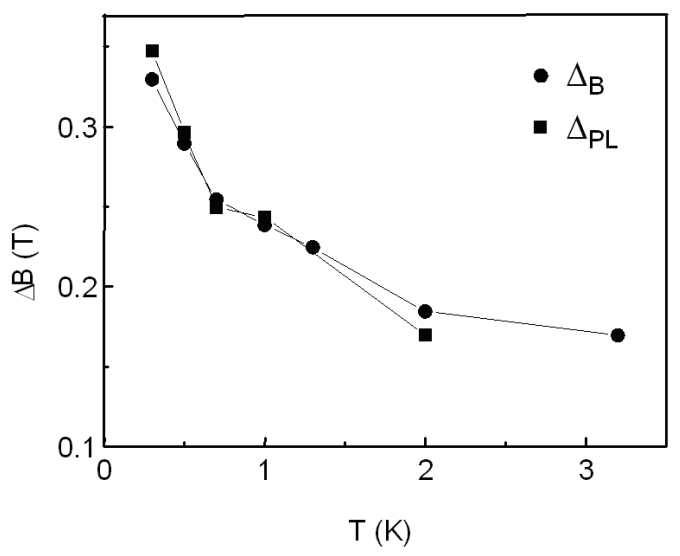

Fig. 8. Temperature dependence of $\Delta_{B}$ and $\Delta_{\mathrm{PL}}$, as defined in Fig. 7.

Similar (but progressive) optical discontinuities in HJs around $\nu=1$ have been reported [5], and explained in terms of a change in the lowest energy state before recombination from an "excitonic" state formed by the electron system and the valence hole above $\nu=1$ to a "free hole" state below $\nu=1$ [18]. It is not clear whether this interpretation can be applied to $\nu=2$, but there are indications that the mechanism underlying the anomalies at both $\nu=1$ and 2 should be similar. In fact, our sample $C$ also displays an abrupt decrease in the $E_{0}$ intensity at $\nu=1$ followed by an "anomaly" of the same characteristics as that shown in Fig. 7 for $\nu=2$.

The nature of the suggested complex state involving the 2DES and the photocreated hole, which would be at the origin of the reported "anomalies", is unknown. It needs further investigation, and in particular theoretical study. The high electron concentration of our samples excludes the formation of charged excitons [11]. A hypothetical complex formed by a small number of missing electrons in the spin-down state of the lowest LL at $\nu<2$ (LL-holes), or missing spin-up electrons at $\nu<1$, and the extended hole, could be considered instead as the origin of the anomalies. Charged complex states of this kind have been proposed [18] for a small number of quasiparticles. Considering sample $B$, the LL-hole density at $\nu=1.8$ is $n_{\mathrm{LLh}}=4.2 \times 10^{10} \mathrm{~cm}^{-2}$. It corresponds to an average inter-LL-hole distance 5 times larger than the magnetic length $l_{\mathrm{B}}\left(l_{\mathrm{B}}=9.3 \mathrm{~nm}\right.$ for $\left.7.5 \mathrm{~T}\right)$. The corresponding parameter $r_{\mathrm{s}}$ (which measures the ratio of the Coulomb energy to the kinetic energy) is $r_{\mathrm{s}}=\left(\pi n_{\mathrm{LLh}}\right)^{-1 / 2}\left(a_{\mathrm{B}}\right)^{-1}=2.7$, where $a_{\mathrm{B}}$ is the effective Bohr radius. Similar values are obtained for samples $A$ and $C$. Thus, the LL-holes can be regarded as an ensemble of independent particles subjected to the Coulomb interaction, which could form the complex state with the valence hole when the filling factor is slightly below 1 or 2 . 


\section{Conclusions}

In summary, we report a narrow FES emission line in the spectrum of a GaAs/AlGaAs HJ containing a high mobility 2DES and a dilute delta-layer of Be. It corresponds to the recombination of electrons in the second confined state with photocreated holes that become trapped at the Be acceptors. The evolution of this line in a perpendicular magnetic field reveals strikingly common characteristics with previous results in high quality samples, which are indicative of a general behavior of 2DES recombination with localized holes. At filling factor 2 an abrupt transfer of emission intensity occurs to recombination of the lowest $E_{0}$-LL electrons with free valence holes, which corresponds to the analogous abrupt onset of this emission in Be-free samples. This emission shows optical "anomalies" consisting of energy and intensity deviations with respect to the single-particle LL behavior. A similar behavior is found also at $\nu=1$ in some samples. The origin of the anomalies is not understood. A tentative explanation is suggested in terms of the formation of a complex state involving the free photocreated hole and the dilute gas of LL-holes. This complex, whose exact nature is unknown, would unbind at higher magnetic fields, when the Fermi level crosses the mobility edge.

\section{Acknowledgments}

This work has been partially supported by the Comunidad Autónoma de Madrid (Project CAM 07N/0064/2001), the Spanish Ministry of Science and Technology (Project Mat 2002-00139) and the COLLECT RTN-project of the EU.

\section{References}

[1] A.J. Turberfield, S.R. Haynes, P.A. Wright, R.A. Ford, R.G. Clark, J.F. Ryan, Phys. Rev. Lett. 65, 637 (1990).

[2] H. Buhmann, W. Joss, K. von Klitzing, I.V. Kukushkin, G. Martinez, A.S. Plaut, K. Ploog, V.B. Timofeev, Phys. Rev. Lett. 65, 1056 (1990).

[3] P. Hawrylak, N. Pulsford, K. Ploog, Phys. Rev. B 46, 15193 (1992).

[4] I.V. Kukushkin, K. von Klitzing, K. Eberl, Phys. Rev. Lett. 82, 3665 (1999); I.V. Kukushkin, J.H. Smet, K. von Klitzing, K. Eberl, Phys. Rev. Lett. 85, 3688 (2000).

[5] R.J. Nicholas, C.C. Chang, V. Zhitomirskiy, in: Optical Properties of Semiconductor Nanostructures, Eds. M.L. Sadowski, M. Grynberg, M. Potemski, Kluwer, Netherlands 2000, p. 33.

[6] C.C. Chang, R.J. Nicholas, Semicond. Sci. Technol. 14, 768 (1999); R.J. Nicholas, D. Kinder, A.N. Priest, C.C. Chang, H.H. Cheng, J.J. Harris, C.T. Foxon, Physica B 249-251, 553 (1998).

[7] J.M. Calleja, H.P. van der Meulen, J. Sanchez, R. Hey, K.J. Friedland, K. Ploog, Solid State Commun. 119, 191 (2001); H.P. van der Meulen, J.M. Calleja, J. Sanchez, R. Hey, K.J. Friedland, K. Ploog, Physica B 319, 339 (2002). 
[8] H.P. van der Meulen, J.M. Calleja, J. Sanchez-Páramo, R. Hey, K.J. Friedland, K. Ploog, in: Proc. 26th Int. Conf. on the Physics of Semiconductors, 26th ICPS, Edinburgh 2002, Eds. A.R. Long, J.H. Davies, Institute of Physics Conference Series, Vol. 171, Inst. of Physics 2003, paper published on included CD.

[9] B.B. Goldberg, D. Heiman, A. Pinczuk, C.W. Tu, A.C. Gossard, J.H. English, Surf. Sci. 196, 209 (1988); D. Heiman, B.B. Goldberg, A. Pinczuk, C.W. Tu, A.C. Gossard, J.H. English, Phys. Rev. Lett. 61, 605 (1988); D. Heiman, B.B. Goldberg, A. Pinczuk, C.W. Tu, A.C. Gossard, J.H. English, Phys. Rev. Lett. 65, 641 (1990).

[10] L. Gravier, M. Potemski, P. Hawrylak, B. Etienne, Phys. Rev. Lett. 80, 3344 (1998).

[11] G. Finkelstein, H. Shtrikman, I. Bar-Joseph, Phys. Rev. Lett. 74, 976 (1995); H. Buhman, L. Mansouri, J. Wang, P.H. Beton, N. Mori, L. Eaves, M. Henini, M. Potemski, Phys. Rev. B 51, 7969 (1995); A. Ron, H.W. Yoon, M.D. Sturge, A. Manassen, E. Cohen, L.N. Pfeiffer, Solid State Commun. 97, 741 (1996); A.J. Shields, M. Pepper, D.A. Ritchie, M.Y. Simmons, G.A.C. Jones, Phys. Rev. B 51, 18049 (1995); Phys. Rev. B 52, R5523 (1995).

[12] K.J. Nash, M.S. Skolnick, M.K. Saker, S.J. Bass, Phys. Rev. Lett. 70, 3115 (1993).

[13] G. Mahan, Many Particle Physics, 2nd ed., Plenum, New York 1990, Sec. 8.3; G. Livescu, D.A.B. Miller, D.S. Chemla, M. Ramaswamy, T.Y. Chang, N. Sauer, A.C. Gossard, J.H. English, IEEE J. Quant. Electron. 24, 1677 (1988); K. Ohtaka, Y. Tanabe, Phys. Rev. B 39, 3054 (1989).

[14] T. Tsuchiya, S. Katayama, T. Ando, Jpn. J. Appl. Phys. 34, 4544 (1995); K. Asano, T. Ando, Physica E 7, 604 (2000).

[15] D.J. Ashen, P.J. Dean, D.T.J. Hurle, J.B. Mullin, A.M. White, J. Phys. Chem. Solids 36, 1041 (1975).

[16] I.V. Kukushkin, K.V. Klitzing, K. Eberl, Phys. Rev. B 55, 10607 (1997).

[17] J.P. Eisenstein, H.L. Stormer, V. Narayanamurti, A.C. Gossard, W. Wiegmann, Phys. Rev. Lett. 53, 2579 (1984).

[18] N.R. Cooper, D.B. Chklovskii, Phys. Rev. B 55, 2436 (1997). 